\title{
Improved split-ubiquitin screening technique to identify surface membrane protein-protein interactions
}

\author{
Daniel Ivanusic ${ }^{1,2}$, Jürgen J. Heinisch ${ }^{3}$, Magdalena Eschricht ${ }^{1}$, Ulrike Laube, and Joachim Denner ${ }^{1}$ \\ ${ }^{1}$ Robert Koch Institute, Berlin, Germany, ${ }^{2}$ Freie Universität Berlin, Berlin, Germany, and ${ }^{3}$ Universität \\ Osnabrück, Osnabrück, Germany
}

BioTechniques 58:63-73 (August 2015) doi 10.2144/000114315

Keywords: protein-protein interactions; split-ubiquitin system; receptors

Supplementary material for this article is available at www.BioTechniques.com/article/114315.

Yeast-based methods are still the workhorse for the detection of protein-protein interactions (PPIs) in vivo. Yeast two-hybrid (Y2H) systems, however, are limited to screening for a specific group of molecules that interact in a particular cell compartment. For this reason, the split-ubiquitin system (SUS) was developed to allow screening of cDNA libraries of full-length membrane proteins for protein-protein interactions in Saccharomyces cerevisiae. Here we demonstrate that a modification of the widely used membrane SUS involving the transmembrane (TM) domain of the yeast receptor Wsc1 increases the stringency of screening and improves the selectivity for proteins localized in the plasma membrane (PM).

During the evolution of life, biological membranes have been critical for establishing barriers, thus allowing molecules in cells to be concentrated and separated. It is essential for living cells that membranes incorporate proteins to manage communication with the environment or perform uptake and export of molecules (1). Membrane proteins are encoded by approximately one third of an organism's genome and have therefore received special attention as putative drug targets. Indeed, more than $60 \%$ of approved drugs target membrane proteins (2). Additional drug targets may also be identified by the discovery of new protein-protein interactions (PPIs) between membrane proteins and their ligands.

The classical $\mathrm{Y} 2 \mathrm{H}$ system as developed by Fields and Song (3) is based on interactions of protein complexes within the nucleus and cannot be used to study the membrane interactions of full-length membrane proteins. A split-ubiquitin system (SUS) has therefore been established (4-6). Yeast-based PPI screens always yield a variable number of false positive interactors because potential interacting proteins are expressed in all cell compartments when testing for PPIs using a cDNA library. These falsely identified interactors are technically sometimes not false, but compartmentalization may prevent some of these identified PPls from ever occurring under physiological conditions. To improve the membrane SUS, we have modified the bait vector PBT3-SUC for type I membrane proteins to allow for selective screening of surface proteins with a higher stringency.

The SUS is based on ubiquitin complementation (7). Ubiquitin is a conserved protein of 76 amino acids that is responsible for marking proteins for degradation by the 265 proteasome (8). Interestingly, ubiquitin can be split into two parts, $\mathrm{N}$-terminal $\left(\mathrm{N}_{\mathrm{ub}}\right)$ ubiquitin and $\mathrm{C}$ terminal ubiquitin $\left(\mathrm{C}_{\mathrm{ub}}\right)$, that are able to re-associate in vivo and form active ubiquitin. This spontaneous process can be inhibited by introduction of a point mutation (113G) into $N_{u b}$, converting the mutated $\mathrm{N}_{\mathrm{ub}} \mathrm{G}$ and the wild-type $\mathrm{C}_{\mathrm{ub}}$ into a composite sensor protein suitable for PPI screening (6). The bait protein of interest is fused to $\mathrm{C}_{\mathrm{ub}}$ and the prey protein to $\mathrm{N}_{\mathrm{ub}} \mathrm{G}$. The reporter protein cassette LexA-VP16 is fused to $C_{u b}$. LexA is a DNA binding domain (9), and VP16 a transcriptional activation domain (10). When a PPI occurs, the reporter protein cassette LexA-VP16 will be cleaved from $\mathrm{C}_{\mathrm{ub}}$ by de-ubiquinating enzymes (ubiquitin-specific proteases) and imported into the nucleus. There it will trigger the expression of typical reporter genes such as HIS3, ADE2 and lacZ and allow for growth on the respective drop-out media or for detection of $\beta$-galactosidase activity (Figure 1).

In contrast to the conventional $\mathrm{Y} 2 \mathrm{H}$ system (3) the SUS does not need to import the entire bait-prey complex into the nucleus; therefore, it allows the use of fulllength membrane proteins for interaction studies in their native environment. However, we assume that the main problem when performing such split-ubiquitin screens is that the transport of the bait proteins to the yeast plasma membrane (PM) is not always guaranteed, for example when viral membrane proteins such as HIV-1 gp41 are studied. In order to avoid this issue, we modified the bait vector PBT3-SUC by substituting the promoter, the signal peptide, and the transmembrane (TM) sequences with those of the yeast cell wall integrity

\section{METHOD SUMMARY}

The widely used split-ubiquitin system was modified using the transmembrane domain of the yeast receptor Wsc1. This increases the screening stringency and enhances the selectivity for those proteins localized in the plasma membrane. 
sensor Wsc1. This well-characterized sensor is predominantly found in the PM of Saccharomyces cerevisiae (11-13). We therefore utilized its ability to localize bait proteins to the PM (where they are available for interaction with prey proteins) for monitoring PPIs.

\section{Material and methods}

Molecular cloning

The bait vector pBT3-SUC-gp41TM ${ }_{\text {gp } 41}$ was cloned using the PCR primer pair DI015/ DI016 (primer sequences are listed in Supplementary Table S1) containing a 5' Sfil linker (GGCCATTACGGCC) and a 3' Sfil linker (GGCCGCCTCGGCC). These primers were used to amplify the gp41 coding sequence (codons 534-709, accession no. AY669735.1) using PfuUltra HF DNA polymerase (Agilent Technologies, Waldbronn, Germany) and the pNL4-3 (14) vector (AIDS Reference and Reagent Program, NIAID, NIH, Bethesda, $\mathrm{MD}$ ) as template. The amplicon was digested with the Sfil restriction enzyme [New England Biolabs (NEB), Frankfurt, Germany] overnight at $50^{\circ} \mathrm{C}$. The digested fragments were ligated with T4 DNA ligase (NEB) into the Sfil-digested vector pBT3-SUC (Dualsystems Biotech AG, Schlieren, Switzerland). The prey vectors pPR3N-CD63 (containing the full-length human CD63 sequence, codons 1-238, accession no. KF998086) and pPR3-NEBP (containing the full-length human emopamil binding protein, EBP, sequence, codons 1-230, accession no. KJ921656) were isolated from split-ubiquitin screens as putative interacting partners of gp41 against a Jurkat cDNA library (Dualsystems Biotech AG). To construct the vector pBT3-WSC1gp41TM $_{\text {gp 41 }}$, plasmid pBT3-SUC-gp41TM gp41 was used to substitute out the promoter and the SUC2 signal sequence for the yeast WSC1 promoter and the Wsc1 signal peptide sequence by a one-step in vivo recombination in yeast. For this purpose, the WSC1 sequences were amplified by PCR from plasmid pSK27 (15) using the oligonucleotides DI017/DI018 as primers. The PCR product was co-transformed with pBT3-SUC-gp41TM ${ }_{\text {gp 41 }}$ linearized at the single $X b a l$ site into the diploid yeast strain DHD5 (16), selecting for leucine prototrophy. Plasmids were isolated from the yeast transformants and amplified in E. coli $\mathrm{DH} 5 \alpha$, selecting for kanamycin resistance. The resulting plasmid was then linearized at the single Pstl site to facilitate another in vivo recombination in yeast. For this purpose, the linearized plasmid was co-transformed with a PCR product obtained using the primers

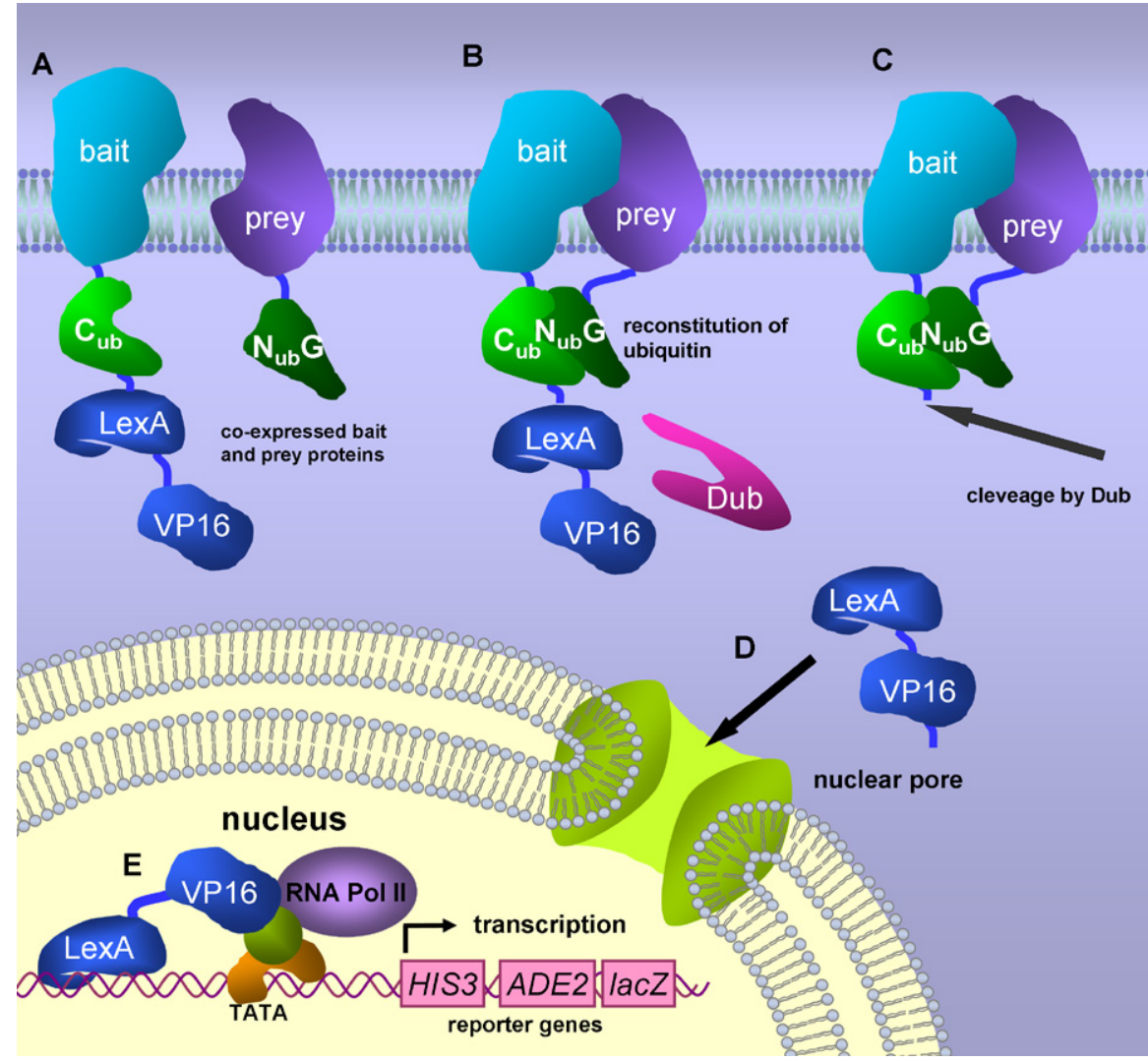

Figure 1. Main components of the membrane-based split-ubiquitin system (SUS). (A) $\mathrm{N}_{\mathrm{ub}} \mathrm{G}$ is not able to associate spontaneously with $\mathrm{C}$ to form a complete ubiquitin. (B) An interaction of the bait and prey proteins allows association of $\mathrm{N}_{\mathrm{ub}} \mathrm{G}$ with $\mathrm{C}_{\mathrm{ub}}$. (C) The reconstituted whole ubiquitin will be recognized by deubiquitinating enzymes (Dub), and the chimeric reporter protein LexA-VP16 is cleaved from the bait protein complex. (D) The reporter protein cassette, LexA-VP16, is transported into the nucleus where (E) LexA binds to the promoter region, and VP16 activates the transcription of typical reporter genes, such as HIS3, ADE2, and lacZ.

DI019/DI020 again on pSK27 as template DNA in order to introduce the sequence encoding the transmembrane domain of Wsc1. Yeast transformants were again selected on medium lacking leucine, plasmids were isolated and amplified in E. coli, and then subjected to restriction and sequence analysis. The resulting vector PBT3-WSC1gp41TM $M_{\text {wsc } 1}$ was used for further investigations. The bait vector PBT3-WSC1-TM (containing only the TM sequence of Wsc1) was generated using the primers DI021/DI022 to amplify the Sfil-5'-TM ${ }_{\text {wsc1 }}$-3'-Sfil fragment from pBT3-WSC1-gp41TM ${ }_{w s c 1}$ as template. The fragment Sfil-5'-TM wsc1 $^{-3}-$ Sfil was cloned into Sfil restricted pBT3-WSC1-gp41TM vector. All cloned constructs were verified by restriction analysis and Sanger sequencing. Oligonucleotides (primers) were obtained from Sigma Aldrich (Steinheim, Germany).

Yeast transformation and yeast growth assay

S. cerevisiae strain NMY51 (Dualsystems Biotech AG) MATa his3 $\Delta 200$ trp1-901 leu2-3,112 ade2 LYS2::(lexAop)4-HIS3 ura3::(lexAop)8-lacZ ade2::(lexAop)8-ADE2 $\mathrm{GAL}$ ) was grown on yeast extract-peptoneadenine-dextrose (YPAD) plates containing $1 \%$ yeast extract (Carl Roth, Karlsruhe, Germany), 2\% peptone (Carl Roth), 2\% glucose (dextrose) (Carl Roth) and 0.004\% adenine sulfate (neoLab, Heidelberg, Germany). Transformed yeast was grown on $0.67 \%$ synthetic yeast medium (Difco Yeast Nitrogen Base w/o Amino Acids; Becton Dickinson and Company, Le pont de Claix, France), 2\% glucose, supplemented with the indicated amino acids W (tryptophan), $\mathrm{L}$ (leucine), $\mathrm{H}$ (histidine), the nucleic acid $\mathrm{A}$ (adenine sulfate), and $10 \times$ single dropout (SD) mix containing $200 \mathrm{mg} / \mathrm{L}$ arginine- $\mathrm{HCl}$, $1500 \mathrm{mg} / \mathrm{L}$ valine, $300 \mathrm{mg} / \mathrm{L}$ isoleucine, 300 mg/L lysine, 200 mg/L methionine, 500 $\mathrm{mg} / \mathrm{L}$ phenylalanine, $2000 \mathrm{mg} / \mathrm{L}$ threonine, 300 mg/L tyrosine, 200 mg/L uracil (all ultra pure L-amino acids and the nucleic acid uracil were obtained from Sigma Aldrich). The final YPAD yeast medium was adjusted to $\mathrm{pH} 6.5$ and the SD medium was adjusted 
A

pBT3-SUC-gp41TM $M_{\text {gp41 }}$

\section{CYC1}

\section{(1)}

$\mathrm{SP}_{\text {suc }}$ gp41 $\mathrm{TM}_{\mathrm{gp} 41} \mathrm{C}_{\mathrm{ub}}$ LexA VP16

pBT3-WSC1-gp41TM gp41 $_{\text {-W }}$

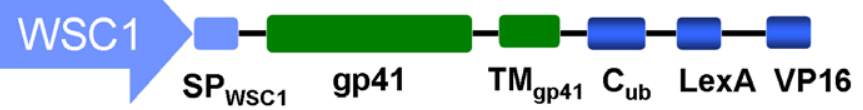

pBT3-WSC1-gp41TM ${ }_{\text {WSC1 }}$

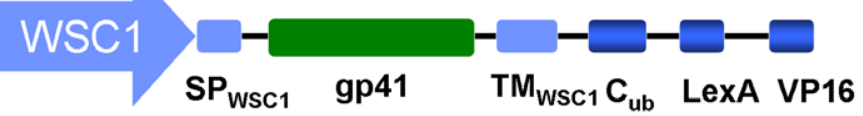

B
(i) 22 aa $\mathrm{TM}_{\mathrm{gp} 41}$
IKLFIMIVGGLVGLRIVFAVLSIVNRVRQG

(ii) 24 aa $\mathrm{TM}_{\mathrm{WSC} 1}$

AIVGGVVGGVVGAVAIALCILLIV

C
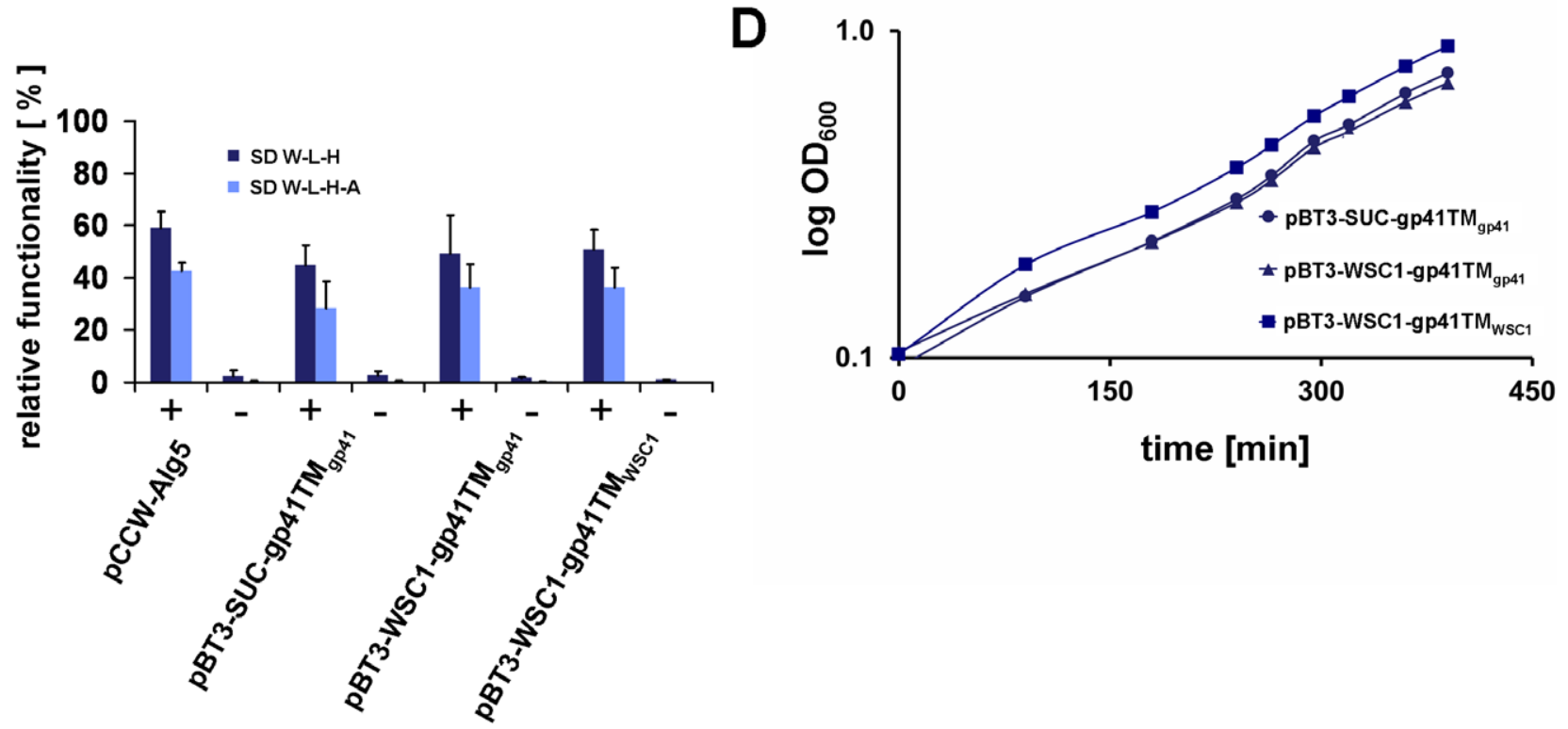

Figure 2. Design and functional characterization of modified bait vectors. (A) Schematic diagrams of unmodified and modified bait vectors. Modified bait vectors contain the WSC1 promotor instead of the CYC1 (cytochrome C-1) promoter. SP: signal peptide from Suc2 or Wsc1; gp41TM $\mathrm{gp}_{1}$ : gp41 containing TM from

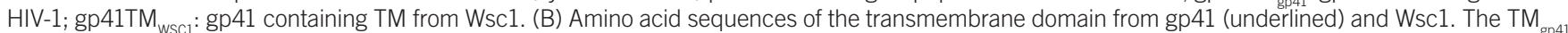
contains the polar amino acid $\mathrm{R}$ (pink arrow) and is flanked by several polar amino acids (red arrows). The complete region of the $\mathrm{TM}_{\mathrm{gp} 41}$ sequence (i) as displayed was exchanged for the $\mathrm{TM}_{\text {wsc1 }}$ sequence by switching the coding sequences (ii). In contrast to $\mathrm{TM}_{\mathrm{gp} 41}, \mathrm{TM}_{\mathrm{wsc1}}$ contains only hydrophobic amino acids. (C) Analysis of the relative functionality in percentage of unmodified vs. modified vectors. NMY51 yeast cells were transformed with the indicated bait vector, along with positive pAI-Alg5 (+) and negative pDL2-Alg5 (-) prey control vectors, and then grown on SD W-L-H (dark blue columns) or SD W-L-H-A (pale blue) selective plates. Error bars represent SD from 3 transformations $(n=3)$. (D) Yeast growth curve: optical density at $600 \mathrm{~nm}\left(\mathrm{OD}_{600}\right)$ is displayed as a function of time (t).

to $\mathrm{pH}$ 5.8. To suppress auto-activity, yeast media were supplemented with 3-amino1,2,4-triazole (3-AT) (Sigma Aldrich) dissolved in pure water. Fourteen g/L agar-agar (Carl Roth) was added to prepare yeast plates. We verified the genotype of S. cerevisiae reporter strain NMY51 by growth analysis on media lacking leucine (SD L), tryptophan (SD W), histidine (SD H), adenine (SD A), and uracil (SD U). Absence of growth on SD L, SD W, and SD U plates was confirmed, and only detached small colonies (around $2 \mathrm{~mm}$ in diameter) were observed on SD $\mathrm{H}$ and SD A plates. These are considered to be background growth due to the slight leakiness of the HIS3 and ADE2 genes. All solutions that could not be autoclaved were filter sterilized using $0.2 \mu \mathrm{m}$ filters (Schleicher
\& Schuell Bioscience, Dassel, Germany). The stock solution of 1M 3-AT was always prepared fresh and was added after the temperature of the yeast media dropped below $50^{\circ} \mathrm{C}$. All transformations to obtain the relative functionality values were performed in the same way. YPAD medium (50 mL) was inoculated with an overnight culture, diluted to an optical density $\left(\mathrm{OD}_{600}\right)$ of 0.15 , 
and the cells were grown to an $\mathrm{OD}_{600}$ of 0.6 before being pelleted and resuspended in $2.5 \mathrm{~mL} \mathrm{H}_{2} \mathrm{O}$. To each transformation tube containing $100 \mu \mathrm{l}$ resuspended yeast cells, we added $300 \mu$ l transformation mix $(2.4 \mathrm{~mL}$ 50\% PEG (MW 3350), 360 ul 1 M Li-acetate, $250 \mu \mathrm{l} 2 \mathrm{mg} / \mathrm{mL}$ salmon sperm DNA (Sigma Aldrich) (15.3 $\mathrm{A}_{260}$ units $/ \mathrm{mg}$, boiled 2 times prior to use), and $1.5 \mu \mathrm{g}$ of prey and bait plasmid DNA. Yeast cells were incubated at $42^{\circ} \mathrm{C}$ for $45 \mathrm{~min}$, pelleted, and resuspended in $150 \mu \mathrm{l} 0.9 \% \mathrm{NaCl}$ (Carl Roth). Yeast cell suspension $(50 \mu \mathrm{l})$ from each transformation was plated onto SD W-L, SD W-L-H, and SD W-L-H-A selective plates. All transformations were performed in triplicate, and yeast colonies were counted to calculate the relative functionality using the formula:

relative functionality $\%=\frac{n_{\text {selective }}}{n_{\text {non-selective }}} \times 100$

( $n_{\text {selective }}=$ number of observed colonies on selective plates, $n_{\text {non-selective }}=$ number of observed colonies on non-selective plates). To calculate the NET $\mathrm{efficiency}$, we subtracted the relative functionality for CD63 on SD W-L-H-A plates from the relative functionality for EBP on SD W-L-H-A plates.
In order to perform pilot screens, we used yeast colonies expressing bait protein, inoculated $100 \mathrm{~mL}$ selective SD L medium with several colonies (minimum 20), and incubated this culture overnight at $30^{\circ} \mathrm{C}$. The next day, we diluted the overnight culture in $200 \mathrm{~mL} S D \mathrm{~L}$ and let the yeast grow overnight before we diluted the yeast culture in $200 \mathrm{~mL} S D \mathrm{~L}$ to achieve an $\mathrm{OD}_{600}$ of 0.15 . This starter culture was incubated with shaking to reach an $\mathrm{OD}_{600}$ of 0.6 (2 cell divisions) and divided into $450-\mathrm{mL}$ aliquots. The aliquots were pelleted, and each was resuspended in $30 \mathrm{~mL} \mathrm{H}_{2} \mathrm{O}$. The yeast cells were pelleted and resuspended in $1 \mathrm{~mL}$ Li-acetate/TE mix (1.1 mL 1M Li-acetate, $1.1 \mathrm{~mL}$ 10x Tris-EDTA, pH 7.5, $7.8 \mathrm{~mL} \mathrm{H}_{2} \mathrm{O}$ ), pelleted again, and resuspended in 600 $\mu \mathrm{L} \mathrm{mL}$ Li-acetate/TE/PEG mix (1.5 mL $1 \mathrm{M}$ Li-acetate, $1.5 \mathrm{~mL}$ 10x Tris-EDTA, pH 7.5, 12 $\mathrm{mL} 50 \%$ PEG (MW 3350). To each $600 \mu \mathrm{L}$ of resuspended cells, we added $7 \mu \mathrm{g}$ library plasmid DNA (Jurkat cDNA library, NubG-X orientation; Dualsystems Biotech AG), 100 ul salmon sperm DNA (2 mg/mL), and 2.5 $\mathrm{mL}$ Li-acetate/TE/PEG mix. Yeast cells were incubated at $30^{\circ} \mathrm{C}$ for 15 min before adding $160 \mu \mathrm{l}$ DMSO (Carl Roth) and continuing the incubation at $42^{\circ} \mathrm{C}$ for $20 \mathrm{~min}$. The yeast cells were incubated for $90 \mathrm{~min}$ at $30^{\circ} \mathrm{C}$ in YPAD medium, pelleted, and resuspended in $4.0 \mathrm{~mL} 0.9 \% \mathrm{NaCl}$. The yeast suspension was plated on SD W-L-H-A $2.5 \mathrm{mM}$ 3-AT using a $32 \times 45 \times 1.5 \mathrm{~cm}$ custom-made stainless-steel plate and incubated at $30^{\circ} \mathrm{C}$. We started to collect colonies on day 5 and picked the last colonies on day 9 after library transformation. Yeast cells were always centrifuged at $700 \times g$ after adding solutions containing Li-acetate, otherwise yeast cells were centrifuged at $2500 \times \mathrm{g}$.

Yeast growth curves were obtained using the transformed NMY51 yeast strain with the indicated bait vectors grown at $30^{\circ} \mathrm{C}$ in SD L liquid media. An overnight culture of transformed yeast was diluted and adjusted to an $\mathrm{OD}_{600}$ of 0.10 . The $\mathrm{OD}_{600}$ was measured at different time points in order to plot the $\mathrm{OD}_{600}$ as a function of time (t).

Proximity ligationassay-based detection of bait proteins within yeast cells

S. cerevisiae cells (NMY51) transformed with modified bait vectors pBT3-WSC1gp41TM $_{\text {gp 41 }}$, pBT3-WSC1-gp41TM wsc1 $_{\text {, }}$, and unmodified pBT3-SUC-gp41TM ${ }_{\text {gp } 41}$, or untransformed NMY51, were grown, starting from an overnight culture, in selective SD L or

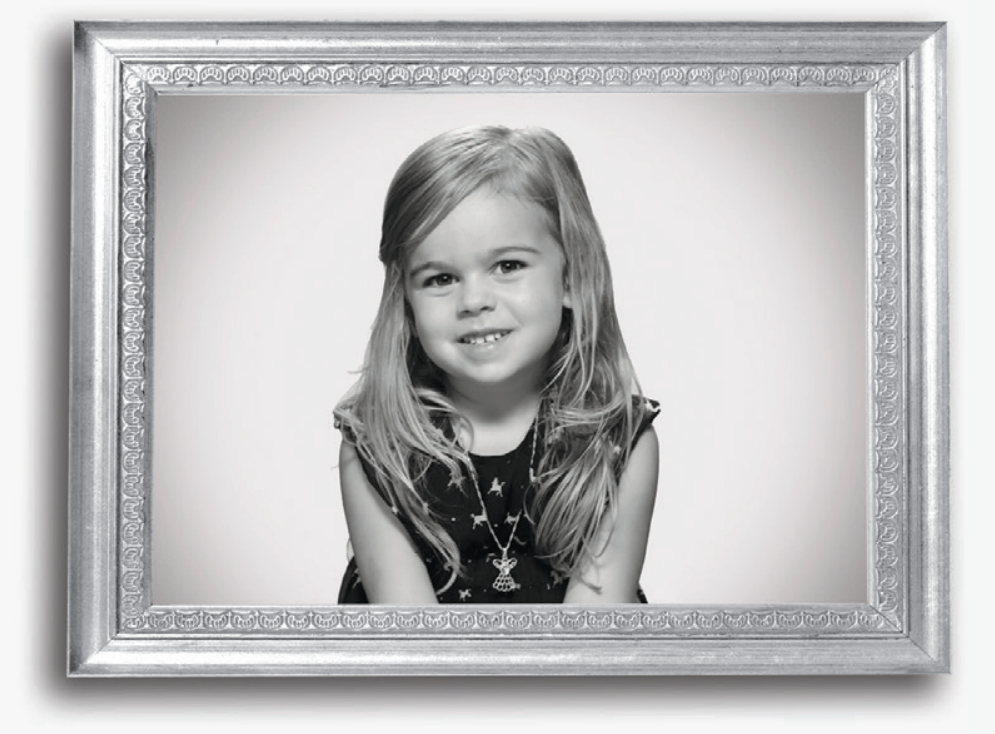

\section{Dear Cancer Researcher,}

I fought cancer once.
Please don't make me do it again.

Lyla Mosser

Cancer Survivor 

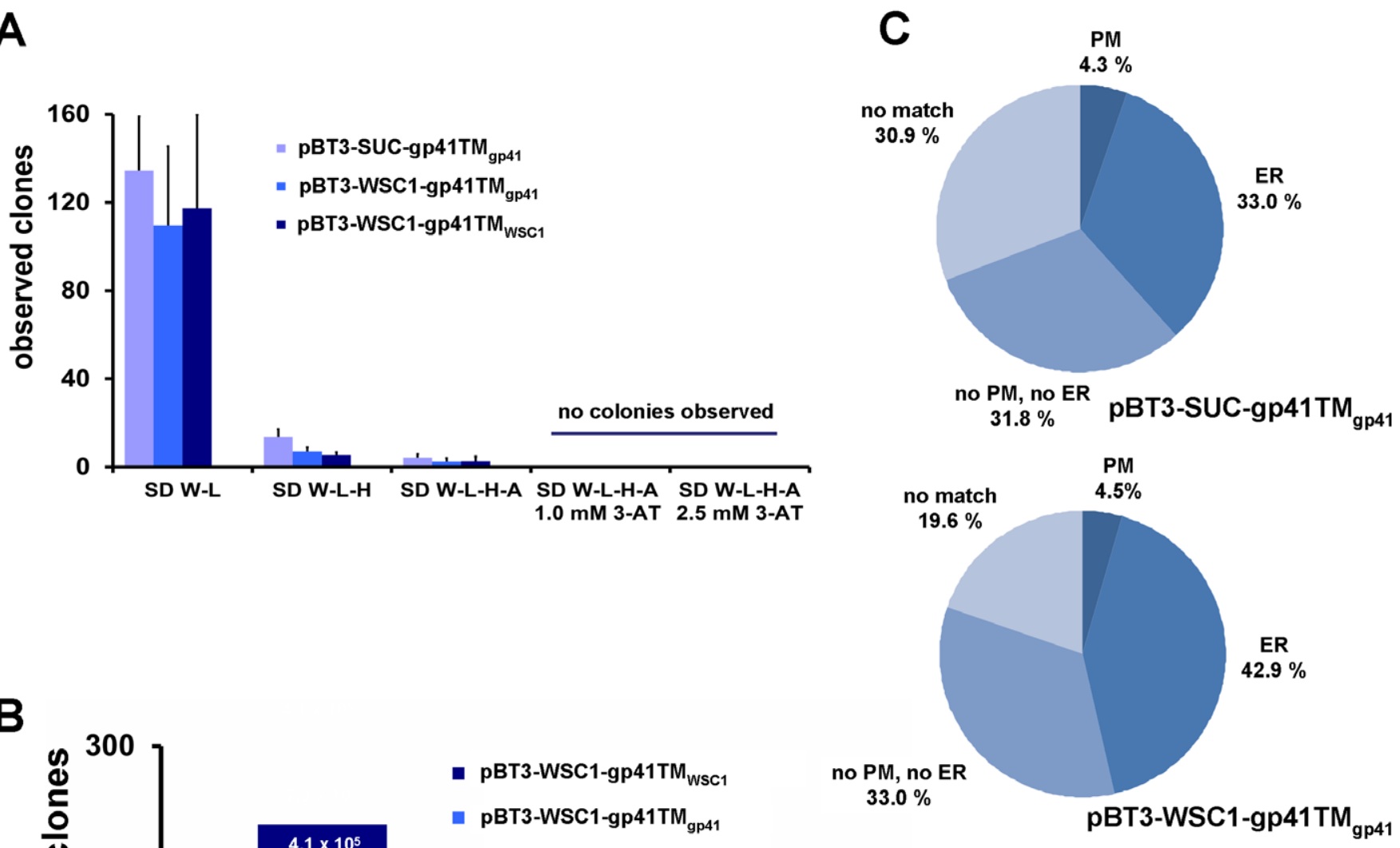

B
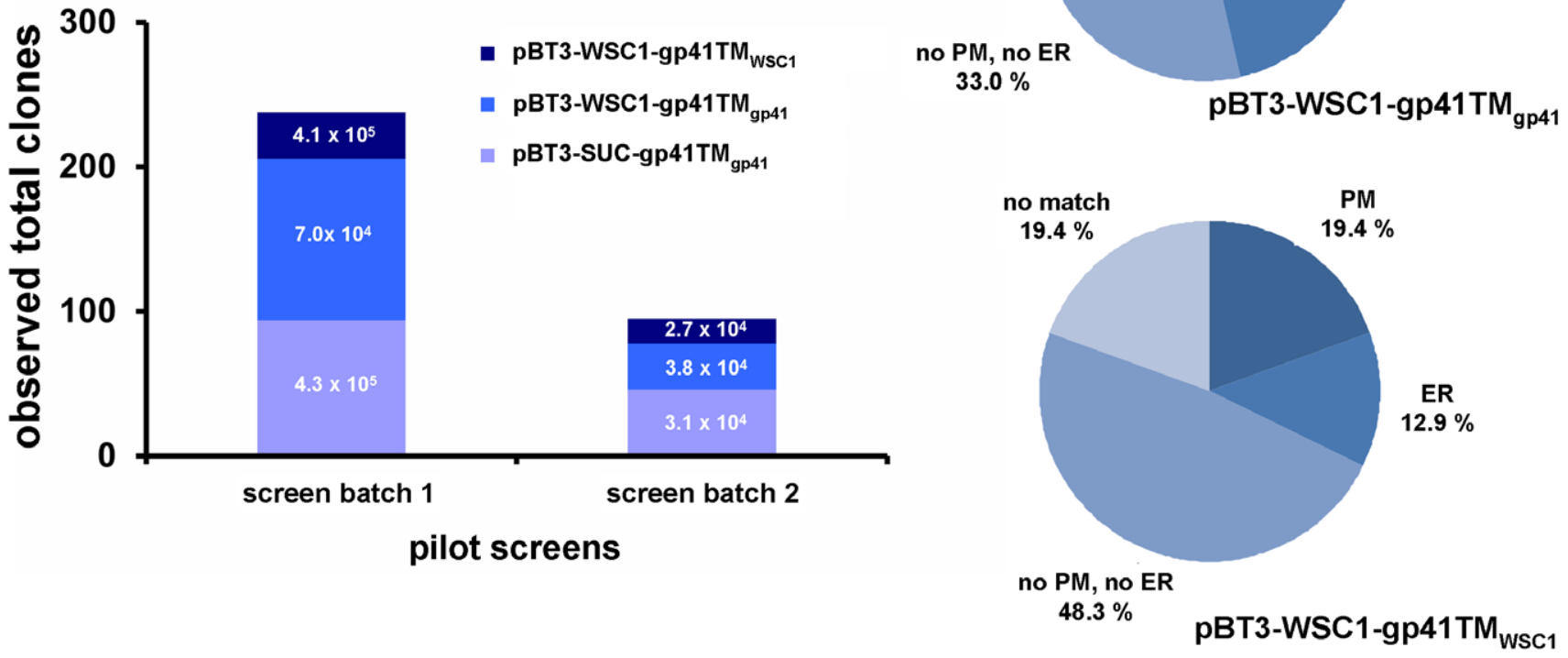

Figure 3. Determination of optimal screening conditions and simulation of library screens. (A) Pilot screen to determine auto-activity mediated by the constructed bait vectors. Yeast cells were transformed with bait vectors and empty prey library vector pPR3-N. Auto-activity was tested under different media conditions as indicated. Error bars: SD $(n=3)$. (B) Simulated library screen performed on SD W-L-H-A, $2.5 \mathrm{mM}$ 3-AT selective plates. Yeast cells expressing baits were transformed with $7 \mu \mathrm{g}$ of a Jurkat cDNA library; the numbers of clones observed are presented relative to the transformation efficiencies (white values within columns). (C) Subcellular localization percentages. Data obtained from pilot screen batch 1 where we achieved the highest transformation efficiencies.

YPAD media at $30^{\circ} \mathrm{C}$ from an $\mathrm{OD}_{600}$ of 0.15 to 0.6. Yeast cells were pelleted and fixed in $2 \%$ paraformaldehyde for $30 \mathrm{~min}$, washed 3 times in sorbitol buffer (1.2 M sorbitol, $100 \mathrm{mM} \mathrm{KH}_{2} \mathrm{PO}_{4}$, pH 6.5) containing 0.5\% 2-mercaptoethanol, then spheroplasted with $100 \mathrm{U} / \mathrm{mL}$ lyticase (Sigma Aldrich) at $30^{\circ} \mathrm{C}$, monitored by light microscopy (Nikon ECLIPSE E600; Nikon, Düsseldorf, Germany). The cells were harvested, washed in sorbitol buffer and dropped on poly-Llysine coated slides (Thermo Scientific, Braunschweig, Germany) within a $2 \mathrm{~cm}^{2}$
PAP-pen (Sigma Aldrich) encircled barrier, excess liquid was removed, and the spheroplasts were air-dried completely before rinsing in $0.2 \%$ Triton-X/PBS for $10 \mathrm{~min}$. The slides were then washed 3 times with sorbitol buffer and blocked with 30\% normal donkey serum (Jackson ImmunoResearch, West Grove, PA) $1 \mathrm{~h}$ at room temperature. After blocking, the spheroplasts were incubated with the primary antibody goat anti-LexA (D-19, sc-1726; Santa Cruz Biotechnology, Heidelberg, Germany) overnight at $4^{\circ} \mathrm{C}$ at a concentration of $5 \mu \mathrm{g} / \mathrm{mL}$. After incubation, the slides were washed extensively 3 times with PBS containing $0.1 \%$ Tween 20. All other steps were performed according to the Duolink II manual, using the proximity ligation assay (PLA) probe anti-goat PLUS and the PLA probe anti-goat MINUS secondary antibodies. To each encircled barrier, $8 \mu \mathrm{L}$ Duolink II mounting medium (containing DAPI for nucleus staining) was added before placing the coverslip on a slide and sealing it with nail polish. All Duolink II detection reagents, mounting media, and PLA probes produced by Olink Bioscience 
(Uppsala, Sweden) were obtained from Sigma Aldrich.

Confocal laser scanning microscopy (CLSM) and image analysis

All images were acquired using a Zeiss LSM 780 confocal laser scanning inverted microscope (Carl Zeiss, Oberkochen, Germany) and a 100x oil immersion objective. PLA signals were detected with ZEISS ZEN smart setup instrument settings for Texas Red analog dye and DAPI.

\section{Determination of clone identity}

We recovered the prey plasmid from each clone grown on SD W-L-H-A screening plates supplemented with $2.5 \mathrm{mM}$ 3-AT. Briefly, clones were taken from a yeast plate (not older than 1 week), stirred into 5 $\mathrm{mL}$ selective media SD W-L-H-A + $2.5 \mathrm{mM}$ $3-A T$, and grown $48 \mathrm{~h}$ at $30^{\circ} \mathrm{C}$. The cells were pelleted, resuspended in $250 \mu$ l buffer P1 (QIAprep Spin Miniprep Kit; Qiagen, Hilden, Germany). Based on the volume of the pellet, $1 / 4$ volume glass beads with a diameter of 0.25-0.50 mm (Carl Roth) was added. The cell wall was cracked by vortexing for $2 \mathrm{~min}$. In accordance to the protocol of the QIAprep Spin Miniprep Kit, prey plasmids were isolated from yeast and retransformed into chemocompetent E. coli XL1-Blue (Stratagene, Heidelberg, Germany). The prey plasmids were isolated from $E$. coli using the same Miniprep kit and sequenced using the forward primer DI023. Identity was analyzed using the Basic Local Alignment Search Tool (BLAST) (http://blast.ncbi.nlm. nih.gov). Information about subcellular localization of identified interacting partners was obtained using the tool COMPARTMENTS (17).

\section{Statistical analysis}

Results are expressed as mean \pm standard deviation (SD). We used a 2-tailed, paired Student's t-test to determine statistical significance, and a $P$-value $<0.05$ was considered as significant between 2 groups.

\section{Results and discussion}

The split-ubiquitin bait vector pBT3-SUCgp41TM $_{\text {gp 41 }}$ was modified using the sequences encoding the signal peptide $\left(\mathrm{SP}_{\text {wSC1 }}\right)$ and the transmembrane domain $\left(\mathrm{TM}_{\text {wsC } 1}\right)$ of Wsc1. In addition, control bait vectors containing only the $\mathrm{SP}_{\text {wsc1 }}$ or only the $\mathrm{TM}_{\mathrm{wSC} 1}$ were generated to analyze the influence of the $\mathrm{TM}_{\mathrm{wsc}}$ on screening efficiency (Figure 2A). The full-length sequences of CD63 or of the emopamil binding protein EBP (also known as sterol isomerase) were used as prey. As baits, an insert of a gp41 construct containing the $\mathrm{N}$-terminal heptad (NHR) and the C-terminal heptad $(\mathrm{CHR})$ region, as well as the $\mathrm{TM}_{\mathrm{gp} 41}$ or $\mathrm{TM}_{\text {wsc1 }}$ sequences were employed (Figure 2B). The modifications also included the removal of the CYC1 promotor and its substitution by the WSC1 promotor.

In order to test the functionality of these modifications and their possible influence on the performance of the bait vectors, a control assay based on the co-transformation of modified bait vectors and control prey vectors pAl-Alg5 (positive control prey vector) and pDL2-Alg5 (negative control prey vector) was used. The co-expression of the positive bait control vector pCCW-Alg5 together with Alg5- $\mathrm{N}_{\mathrm{ub}}$ I from pAl-Alg5 results in a re-association of split-ubiquitin by the strong affinity of wild-type $\mathrm{N}_{u b}$ I for $\mathrm{C}_{\mathrm{ub}}$. This will activate reporter genes, allowing yeast colonies to grow on low SD W-L-H and on highly selective SD W-L-H-A plates. Yeast growth on SD W-L-H and SD W-L-H-A plates therefore indicates that the bait is functional

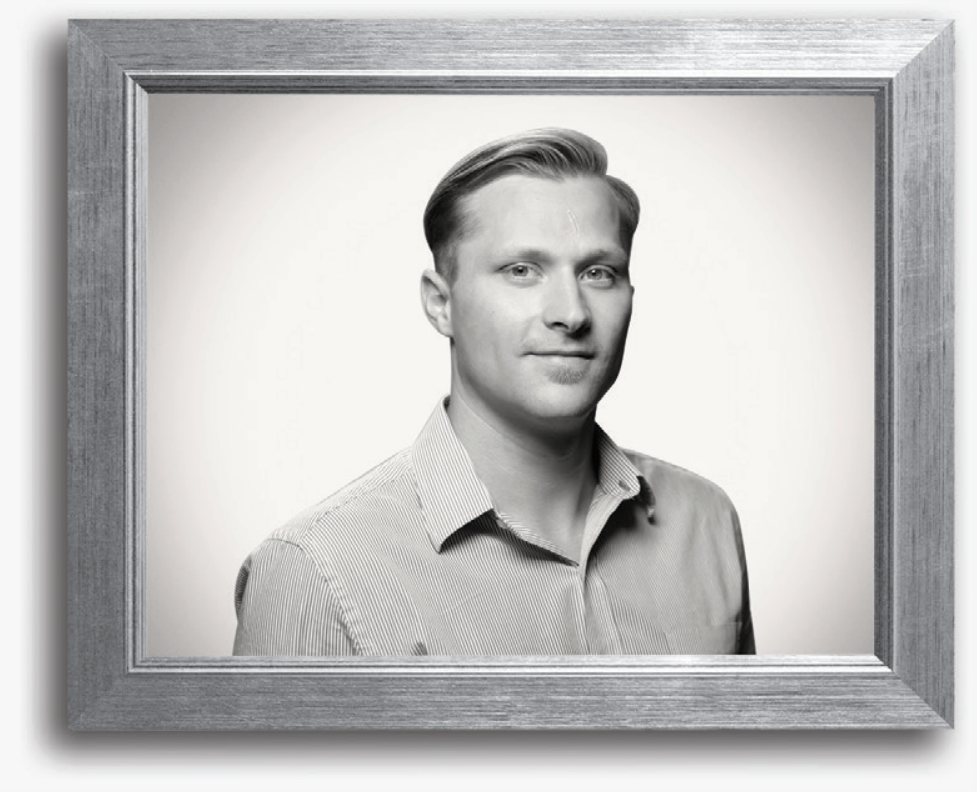

Dear Cancer Researcher,

People call me a hero.

Your work could make you one.

Frank Caruso

Cancer Survivor 
A

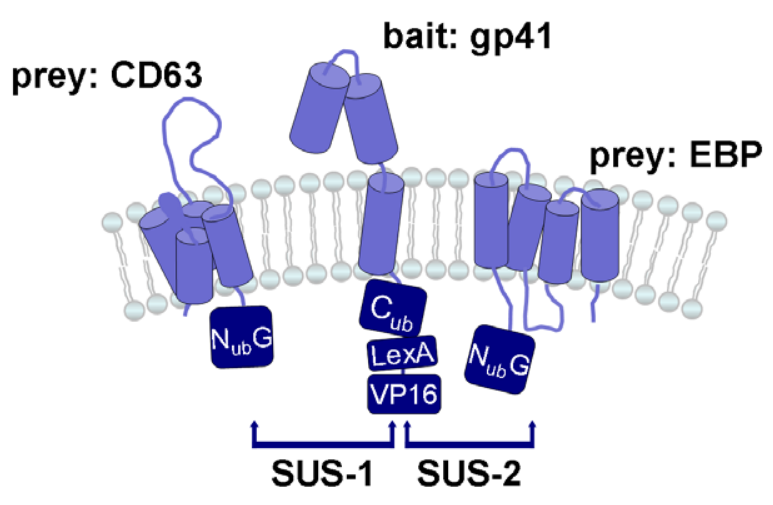

C
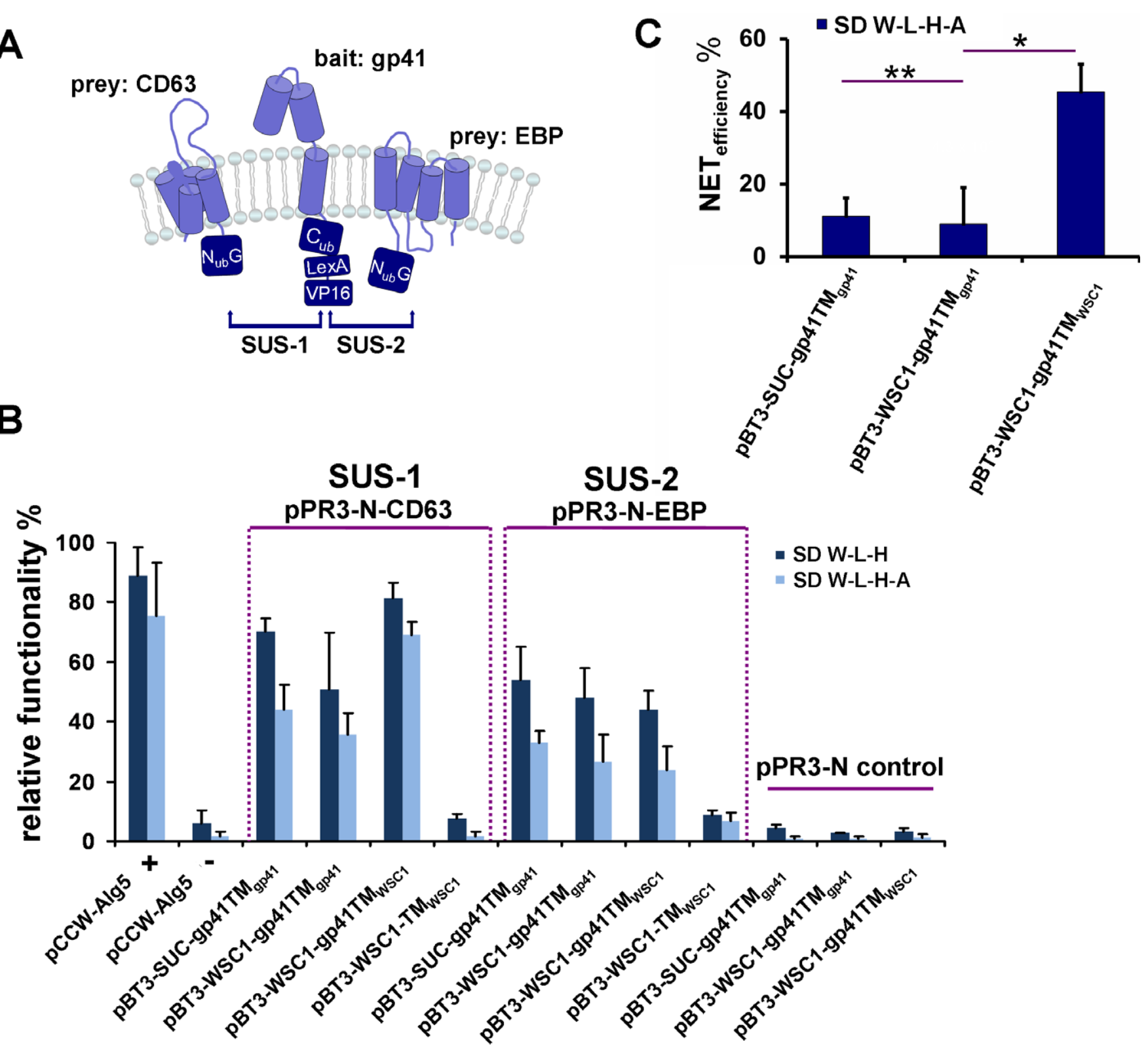

D

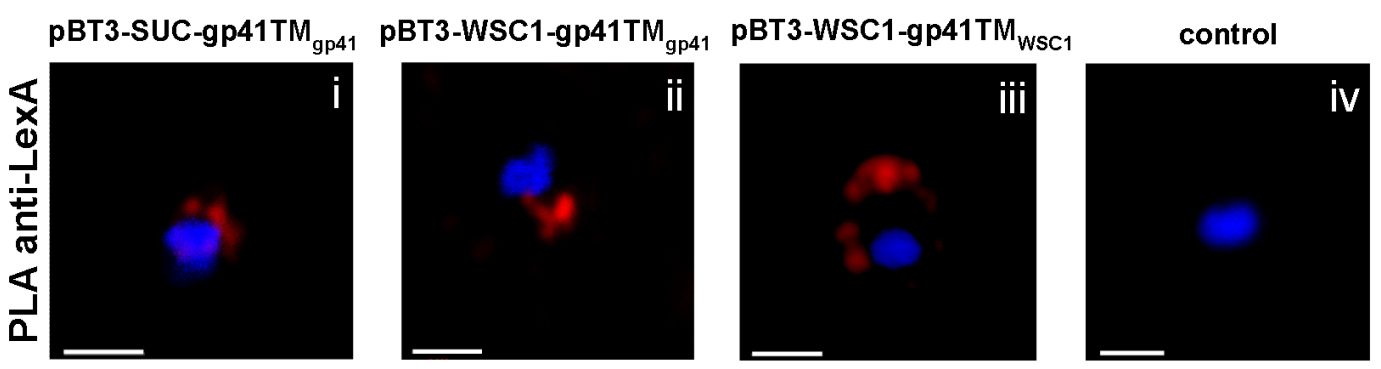

Figure 4. Investigation of selectivity using modified and unmodified bait vectors. (A) Diagram of the experiments determining the selectivity property of modified bait vectors vs. the unmodified bait vector. (B) Sequential determination of percentage growth on SD W-L-H and SD W-L-H-A selective media. (C) $\mathrm{NET}_{\text {efficiency }}$ (difference of the percentage growth on SD W-L-H-A selective plates between CD63 and EBP). Increased selectivity of the bait against CD63 using $\mathrm{TM}_{\mathrm{WSc} 1}$ in the bait vector systems. All error bars: $\mathrm{SD}(n=3)$. ${ }^{*} P=0.008$; ${ }^{*} P=0.765$. (D) Confocal images of proximity ligation assay (PLA) signals showing the localization of bait proteins in spheroplasts of $S$. cerevisiae NMY51. PLA performed against the LexA epitope using goat anti-LexA antibody. Images show signals from PLA experiments (red) against the LexA epitope using S. cerevisiae (NMY51) spheroplasts transformed with (i) pBT3-SUC-gp41TM ${ }_{\text {gp41 }}$ (ii) pBT3-

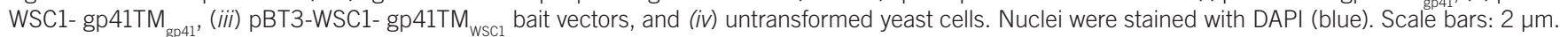


in the SUS. In contrast, when pCCW-Alg5 is co-expressed with DL2-Alg5, the yeast transformants did not grow on selective plates because the mutated $N_{u b} G$ is not able to re-associate spontaneously with $\mathrm{C}_{\mathrm{ub}}$.

To quantify the performance of modified bait vectors, the percentage of growth on selective plates SD W-L-H and plates SD W-L-H-A versus non-selective plates SD W-L, which only reflect the transformation efficiency, was analyzed. More than $20 \%$ of the bait vectors conferred growth, which is in the range recommended by the Dualsystems manual to evaluate functionality. In fact, the functionality values for the vectors pBT3-WSC1-gp41TM gp 41 $_{1}$ and pBT3-WSC1gp $41 \mathrm{TM}_{\text {wsc } 1}$ were over $36 \%$ on SD W-L-H-A plates (Figure 2C). This indicated that all constructs are well-expressed and that the $\mathrm{C}_{\mathrm{ub}}$ was sufficiently exposed to be able to interact with the $\mathrm{N}_{\mathrm{ub}} \mathrm{l}$.

The negative controls showed that $\mathrm{C}_{\mathrm{ub}}$ did not interact with $\mathrm{N}_{\mathrm{ub}} \mathrm{G}$ and that the reporter genes are not expressed sufficiently to allow growth on selective plates. The controls using the bait vector pCCW-Alg5 with the prey vectors pAl-Alg5 (positive control) and pDL2-Alg5 (negative control) showed functionalities comparable to that of the modified vectors. These controls also confirmed that the baits used have the same orientation (outside- $\mathrm{x}-\mathrm{C}_{\mathrm{ub}}$-inside) as the positive control prey Al-Alg5 (outside$x-N_{\mathrm{ub}}$-inside). A possible switch to type II proteins was therefore excluded (18).

We further analyzed possible toxic effects on transformed yeast cells that may be caused by these modifications. The monitoring of yeast growth by $\mathrm{OD}_{600}$ affirmed that all transformed yeast cells cultured in non-selective media SD L (selective for yeast cells transformed with bait vectors) reached exponential growth, comparable to yeast cells transformed with the unmodified bait vector pBT3-SUC-gp41TM $\mathrm{gp} 41_{\text {(Figure 2D). }}$. These data indicate that neither the substitution of the CYC1 promoter nor the introduction of $\mathrm{SP}_{\text {wSC1 }}$ and $\mathrm{TM}_{\text {wSC1 }}$ exerted a negative effect on the bait protein expression or were toxic for S. cerevisiae.

Optimized split-ubiquitin screening requires that the screening stringency be determined by supplementing media with 3-AT. This compound competitively inhibits the enzyme imidazole-glycerol-phosphate dehydratase encoded by the HIS3 reporter gene $(19,20)$. HIS3 is known to be subject to leaky expression (21), and therefore, the yeast growth should be tested on selective media for auto-activity, and if necessary, this should be supplemented with 3-AT to increase stringency and reduce the number of false positive interactors. The screens were carried out utilizing the $S$. cerevisiae reporter strain NMY51 with HIS3 and ADE2 genes under the control of the lexA operator. The modified bait vectors pBT3-WSC1gp41TM $_{\text {gp 41 }}$ and pBT3-WSC1-gp41TM wsC1 $_{\text {-W }}$ and the unmodified pBT3-SUC-gp41TM bait vector were co-transformed with the empty library prey vector pPR3-N. The optimal concentration of 3-AT suitable to suppress auto-activation (also known as self-activation) was determined by titration. The transformed yeast cells were streaked onto the selective plates SD W-L-H and SD W-L-H-A containing the indicated concentrations of 3-AT. The optimal screen stringency was assessed by the absence of growing yeast colonies. All bait vectors tested for auto-activity lacked yeast growth at a 3-AT concentration of $1.0 \mathrm{mM}$ on SD W-L-H-A (Figure 3A). Based on this observation, we concluded that the modifications introduced as shown in Figure 2A did not increase the auto-activity mediated by the bait constructs.

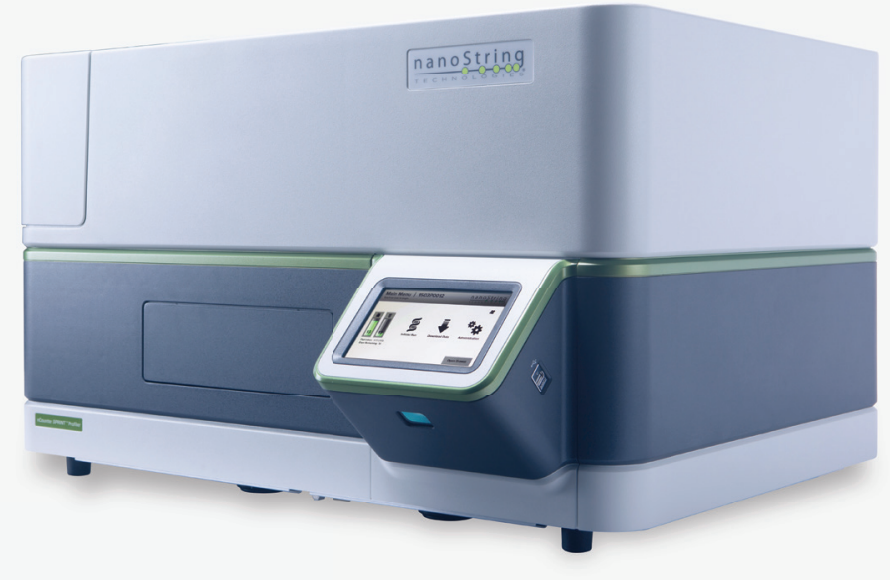

\section{nCounter SPRINT ${ }^{\text {TM }}$ Profiler THE REVOLUTION HAS BEGUN}

\author{
The end of RT-qPCR and targeted RNA-Seq? \\ Even cancer itself? Explore the new nCounter \\ SPRINTProfiler and see why our claims are being \\ widely embraced. Offering exceptional precision \\ and flexibility, nCounter SPRINT enables direct \\ single-molecule counting - thus accelerating \\ your cancer research beyond the limitations of \\ qPCR and NGS.
}

RESEARCH USE ONLY. Not for use in diagnostic procedures. 
In order to determine how the modifications affect the clone yields, a simulated split-ubiquitin screen with modified versus unmodified bait vectors was performed. For this purpose, we co-transformed each bait vector with $7 \mu \mathrm{g}$ of a cDNA Jurkat cell library ( $\mathrm{N}_{\mathrm{ub}} \mathrm{G}-\mathrm{x}$ orientation), which comprises a complexity of $9 \times 10^{6}$ independent clones. The transformed yeast cells were streaked onto selective SD W-L-H-A plates supplemented with a higher 3-AT concentration of $2.5 \mathrm{mM}$ to avoid putative weak-interacting preys. Interestingly, the transformation with the cDNA library using the modified bait vector pBT3-WSC1-gp41TM wsc1 $_{\text {yielded }}$ the lowest amount of colonies on selective plates SD W-L-H-A supplemented with 2.5 mM 3-AT (Figure 3B). Detecting PPIs with the modified bait vector PBT3-WSC1gp41TM $M_{\text {wsc } 1}$ therefore seems to enhance the stringency in our split-ubiquitin screens. The introduction of the TM domain from Wsc1 apparently changed the number of observable clones grown on screening plates. In two screen batches, we consistently observed a higher number of clones on selective plates when we used the bait vectors without $\mathrm{TM}_{\mathrm{wsC1}}$, which may indicate that a large number of membrane proteins are already interacting with the original constructs in the endoplasmic reticulum (ER), rather than in the plasma membrane. A split-ubiquitin screen is virtually impossible to follow up when it yields an excessive number of clones. Therefore, the introduction of the $\mathrm{TM}_{\text {wsc1 }}$ introduced a higher screening selectivity, which allows prey proteins to be detected only where they naturally accumulate. To study this, prey plasmids were isolated and their identities were determined by sequencing and BLAST analysis (Supplementary Tables S2-S4). The subcellular localization of the identified proteins was determined using the tool COMPARTMENTS. Using the bait vectors pBT3-SUCgp41TM $_{\text {gp } 41}$ and pBT3-WSC1-gp41TM ${ }_{\text {gp } 41}$, the identified prey proteins localized in the PM were only found $4.3 \%-4.5 \%$ of the time, but using the vector pBT3-WSC1-gp41TM wsc1 $_{\text {I }}$ as bait, $19.4 \%$ of the prey proteins were localized in the PM (Figure 3C). For all bait constructs, a substantial number of putative interacting partners were identified with a localization not in the PM or ER (Figure 3C). This number was not reduced by using the modified vector pBT3-WSC1-gp41TM ${ }_{\text {wsc1 }}$, but rather increased to $48.3 \%$.

To quantify the selectivity for surfacelocalized membrane proteins, CD63 with its well-known localization in mammalian cells was used as a reference protein. CD63 is a tetraspanin that contains four transmembrane domains (a 4TM protein). This protein is predominantly localized in the plasma membrane $(22,23)$, and we identified it as an interacting partner of gp41 (24). In addition to CD63, another 4TM protein, the emopamil binding protein EBP (sterol isomerase), was also identified in earlier split-ubiquitin screens as a frequent interaction partner of gp41. Furthermore, EPB was also found to interact with other bait constructs such as VKORC1 (25). In contrast to CD63, EBP is localized in the ER membrane but not in the PM $(26,27)$. These two proteins were used to validate the selectivity of the modified bait vectors for prey proteins localized either in the ER or PM (Figure 4A). To quantify the differences between the modified and the unmodified bait vector system in the context of selectivity for proteins localized in the PM, prey vectors containing CD63 or EBP inserts were co-transformed with the bait vectors. The co-expression of prey CD63 and bait gp41 yielded significantly higher colony numbers on selective plates than co-expression of prey EBP with the gp41 bait construct (Figure 4B). We could also exclude the possibility that the $\mathrm{TM}_{\mathrm{wsc}}$ causes unspecific self-activation of the sensor protein $\mathrm{N}_{\mathrm{ub}} \mathrm{G} / \mathrm{C}_{\mathrm{ub}}$ since the transformations with the vector PBT3-WSC1-TM wsc1 $_{\text {I }}$ (containing only the transmembrane domain of Wsc1) showed no significant growth on the selective plates. Furthermore, the difference between the relative functionality (assessed on SD W-L-H-A plates) of CD63 and EBP was calculated using the data set from Figure 4B. The calculated difference between the functionality of CD63 and EBP demonstrated clearly that (i) the interaction affinity of gp41 for CD63 protein was higher than that of EBP, and (ii) that the relative functionality of the bait vector PBT3-WSC1gp41TM $_{\text {wsc } 1}$ containing the $\mathrm{TM}_{\text {wsc } 1}$ was increased at least by a factor of four compared with the unmodified bait vectors pBT3-SUC-gp41TM ${ }_{\text {gp } 41}$ and pBT3-WSC1gp41TM $_{\text {gp } 41}$. These data confirmed that the Wsc1 transmembrane-anchored gp41 construct displayed an increased affinity to the prey protein localized in the PM.

The $\mathrm{TM}_{\mathrm{gp} 41}$ contains polar charged amino acids and therefore it is possible that the transmembrane domain-dependent routing of proteins containing this transmembrane domain to the PM in S. cerevisiae is impaired because the transmembrane domains of typical transmembrane proteins sorting to the PM have a higher content of hydrophobic amino acids and are 24 amino acids in length (28). In the case of the $\mathrm{TM}_{\mathrm{gp} 41}$ from HIV-1, the transmembrane region contains 22 residues, but more importantly, polar charged amino acid residues are included in the TM domain, which itself is further flanked by polar charged residues (Figure 2B). This explains why bait proteins in S. cerevisiae fused with the SUC2 signal sequence in conjunction with an inefficient transmembrane domain were retained in the ER membrane. The SUC2 signal sequence originally directs the secretion of yeast invertase, and heterologous proteins with this signal sequence frequently become stuck in the ER (29). Therefore, the combination of a viral TM that reaches the PM with low efficiency and a signal sequence from proteins that frequently retain proteins at the ER is definitely not optimal to direct bait proteins to screen for receptors localized in their native location. The screening conditions were improved by the introduction of $\mathrm{TM}_{\text {wsc1 }}$, and a higher efficiency was achieved when these designed proteins were used as baits.

Finally, we examined the localization in yeast cells expressing unmodified and modified bait proteins. We used the proximity ligation assay (PLA) technique for this to detect highly specific and sensitive (30-31) weakly expressed proteins driven by the CYC1 (32) or WSC1 (13) promoter. The single recognition PLA analysis performed against the LexA epitope using one primary polyclonal antibody revealed a localization for the bait proteins expressed by the vector pBT3-WSC1-gp41TM wsc1 $_{\text {}}$ at the cell periphery only. In contrast, the bait proteins expressed by the vectors pBT3-WSC1-gp41TM ${ }_{\text {gp } 41}$ and pBT3-SUCgp41TM $_{\text {gp41 }}$ were found in the perinuclear space (Figure 4D). We concluded that Wsc1-TM-anchored gp41 bait proteins are targeted to the cell periphery where they accumulate and are able to achieve close proximity to their interacting partner in the same compartment.

Here we present the modification of a membrane-based split-ubiquitin system (SUS) to improve the screening stringency for surface membrane proteins. Our modified SUS is aimed at the study of host-pathogen and receptor-ligand interactions, as well as PPIs involved in cell-cell communication. We modified the widely used split-ubiquitin bait vector PBT3-SUC and showed that the transmembrane 
domain of Wsc1 increases the selectivity of type I membrane proteins such as gp41 to interact with the tetraspanin CD63. By using modified bait vectors, the experiments presented here helped to prevent split-ubiquitin screens from yielding too many candidate clones. We realize that this is only an initial application of our method and that it can be specifically adapted to other needs (e.g., to direct the bait protein to the mitochondrial membrane in order to search for binding proteins localized there). Screens with increased specificity can be therefore performed, which will yield lower numbers of false positive clones and facilitate the identification of putative interaction candidates for further research.

\section{Author contributions}

D.I. designed and performed the experiments and analyzed the data. J.J.H. designed and cloned the modified vectors. U.L. performed the yeast transformations and isolated the prey library vectors. The manuscript was written by D.I., J.J.H., M.E., and J.D. All authors read and approved the manuscript.

\section{Acknowledgments}

This work was supported by the Peter and Traudl Engelhorn Foundation with a grant to Daniel Ivanusic and JungStiftung für Wissenschaft und Forschung, Hamburg,Germany. Work in the laboratory of Jürgen J. Heinisch was financed by a grant from the Deutsche Forschungsgemeinschaft within the framework of the SFB944. We are pleased to thank Steve Norley for critical reading of the manuscript.

\section{Competing interests}

The authors declare no competing interests.

\section{References}

1. Lombard, J., P. Lopez-Garcia, and D. Moreira. 2012. The early evolution of lipid membranes and the three domains of life. Nat. Rev. Microbiol. 10:507-515.

2. Yildirim, M.A., K.-I. Goh, M.E. Cusick, A.-L. Barabasi, and M. Vidal. 2007. Drug-target network. Nat Biotech. 25:1119-1126.

3. Fields, S. and O. Song. 1989. A novel genetic system to detect protein-protein interactions. Nature 340:245-246.

4. Brückner, A., C. Polge, N. Lentze, D. Auerbach, and U. Schlattner. 2009. Yeast two-hybrid, a powerful tool for systems biology. Int. J. Mol. Sci. 10:2763-2788.

5. Stagljar, I., C. Korostensky, N. Johnsson, and S. te Heesen. 1998. A genetic system based on split-ubiquitin for the analysis of interactions between membrane proteins in vivo. Proc. Natl. Acad. Sci. USA 95:5187-5192.

6. Johnsson, N. and A. Varshavsky. 1994. Split ubiquitin as a sensor of protein interactions in vivo. Proc. Natl. Acad. Sci. USA 91:1034010344.

7. Stynen, B., H. Tournu, J. Tavernier, and P. Van Dijck. 2012. Diversity in genetic in vivo methods for protein-protein interaction studies: from the yeast two-hybrid system to the mammalian split-luciferase system. Microbiol. Mol. Biol. Rev. 76:331-382.

8. Hershko, A. and A. Ciechanover. 1992. The ubiquitin system for protein degradation. Annu. Rev. Biochem. 61:761-807.

9. Thliveris, A.T. and D.W. Mount. 1992. Genetic identification of the DNA binding domain of Escherichia coli LexA protein. Proc. Natl. Acad. Sci. USA 89:4500-4504.

10. Wu, T.J., G. Monokian, D.F. Mark, and C.R. Wobbe. 1994. Transcriptional activation by herpes simplex virus type 1 VP16 in vitro and its inhibition by oligopeptides. Mol. Cell. Biol. 14:3484-3493.

11. Dupres, V., D. Alsteens, S. Wilk, B. Hansen, J.J. Heinisch, and Y.F. Dufrene. 2009. The yeast Wsc1 cell surface sensor behaves like a nanospring in vivo. Nat. Chem. Biol. 5:857-862.

12. Heinisch, J.J., V. Dupres, S. Wilk, A. Jendretzki, and Y.F. Dufrene. 2010. Singlemolecule atomic force microscopy reveals clustering of the yeast plasma-membrane sensor Wsc1. PLoS ONE 5:e11104.

13. Lodder, A.L., T.K. Lee, and R. Ballester. 1999. Characterization of the Wsc1 protein, a putative receptor in the stress response of Saccharomyces cerevisiae. Genetics 152:1487-1499.

14. Adachi, A., H.E. Gendelman, S. Koenig, T. Folks, R. Willey, A. Rabson, and M.A. Martin. 1986. Production of acquired immunodeficiency syndrome-associated retrovirus in human and nonhuman cells transfected with an infectious molecular clone. J. Virol. 59:284-291.

15. Wilk, S., J. Wittland, A. Thywissen, H.P. Schmitz, and J.J. Heinisch. 2010. A block of endocytosis of the yeast cell wall integrity sensors Wsc1 and Wsc2 results in reduced fitness in vivo. Mol. Genet. Genomics 284:217229

16. Kirchrath, L., A. Lorberg, H.P. Schmitz, U. Gengenbacher, and J.J. Heinisch. 2000. Comparative genetic and physiological studies of the MAP kinase Mpk1p from Kluyveromyces lactis and Saccharomyces cerevisiae. J. Mol. Biol. 300:743-758.

17. Binder, J.X., S. Pletscher-Frankild, K. Tsafou, C. Stolte, S.I. O'Donoghue, R. Schneider, and L.J. Jensen. 2014. COMPARTMENTS: unification and visualization of protein subcelIular localization evidence. Database (Oxford) 2014:bau012.

18. Shao, S. and R.S. Hegde. 2011. Membrane protein insertion at the endoplasmic reticulum. Annu. Rev. Cell Dev. Biol. 27:25-56.

19. Klopotowski, T. and A. Wiater. 1965. Synergism of aminotriazole and phosphate on the inhibition of yeast imidazole glycerol phosphate dehydratase. Arch. Biochem. Biophys. 112:562-566.

20. Struhl, K. and R.W. Davis. 1977. Production of a functional eukaryotic enzyme in Escherichia coli: cloning and expression of the yeast structural gene for imidazole-glycerolphosphate dehydratase (his3). Proc. Natl. Acad. Sci. USA 74:5255-5259.

21. Van Criekinge, W. and R. Beyaert. 1999 Yeast Two-Hybrid: State of the Art. Biol. Proced. Online 2:1-38.

22. Mantegazza, A.R., M.M. Barrio, S. Moutel, L. Bover, M. Weck, P. Brossart, J.L. Teillaud, and J. Mordoh. 2004. CD63 tetraspanin slows down cell migration and translocates to the endosomal-lysosomal-MIICs route after extracellular stimuli in human immature dendritic cells. Blood 104:1183-1190.

23. Kannan, K., S.G. Divers, A.A. Lurie, R. Chervenak, M. Fukuda, and R.F. Holcombe. 1995. Cell surface expression of lysosomeassociated membrane protein-2 (lamp2) and CD63 as markers of in vivo platelet activation in malignancy. Eur. J. Haematol. 55:145-151.

24. Ivanusic, D., M. Eschricht, and J. Denner 2014. Investigation of membrane proteinprotein interactions using correlative FRET-PLA. Biotechniques 57:188-191.

25. Schaafhausen, A., S. Rost, J. Oldenburg, and C.R. Muller. 2011. Identification of VKORC1 interaction partners by split-ubiquitin system and coimmunoprecipitation. Thromb. Haemost. 105:285-294.

26. Moebius, F.F., G.G. Burrows, J. Striessnig, and H. Glossmann. 1993. Biochemical characterization of a 22-kDa high affinity antiischemic drug-binding polypeptide in the endoplasmic reticulum of guinea pig liver: potential common target for antiischemic drug action. Mol. Pharmacol. 43:139-148.

27. Dussossoy, D., P. Carayon, S. Belugou, D. Feraut, A. Bord, C. Goubet, C. Roque, H. Vidal, et al. 1999. Colocalization of sterol isomerase and sigma(1) receptor at endoplasmic reticulum and nuclear envelope level. Eur. J. Biochem. 263:377-386.

28. Rayner, J.C. and H.R. Pelham. 1997. Transmembrane domain-dependent sorting of proteins to the ER and plasma membrane in yeast. EMBO J. 16:1832-1841.

29. Emr, S.D., I. Schauer, W. Hansen, P. Esmon, and R. Schekman. 1984. Invertase beta-galactosidase hybrid proteins fail to be transported from the endoplasmic reticulum in Saccharomyces cerevisiae. Mol. Cell. Biol. 4:2347-2355.

30. Söderberg, O., K.J. Leuchowius, M. KamaliMoghaddam, M. Jarvius, S. Gustafsdottir, E. Schallmeiner, M. Gullberg, J. Jarvius, and U. Landegren. 2007. Proximity ligation: a specific and versatile tool for the proteomic era. Genet. Eng. (N. Y.) 28:85-93.

31. Schallmeiner, E., E. Oksanen, O. Ericsson, L. Spangberg, S. Eriksson, U.H. Stenman, K. Pettersson, and U. Landegren. 2007. Sensitive protein detection via triple-binder proximity ligation assays. Nat. Methods 4:135-137.

32. Mumberg, D., R. Muller, and M. Funk. 1995. Yeast vectors for the controlled expression of heterologous proteins in different genetic backgrounds. Gene 156:119-122.

Received 17 June 2014; accepted 26 June 2015.

Address correspondence to Joachim Denner, Robert Koch Institute, Berlin, Germany. E-mail: DennerJ@rki.de

To purchase reprints of this article, contact: biotechniques@fosterprinting.com 